\title{
Ichthyofauna of the humid forest enclaves in the tablelands of Ibiapaba and Araripe, Northeastern Brazil
}

Carlos Alberto de Sousa Rodrigues-Filho', Ronaldo César Gurgel-Lourenço², Luis Artur Valões Bezerra ${ }^{3}$, Wallace Alves de Sousa ${ }^{4}$, Danielle Sequeira Garcez ${ }^{4}$, Sergio Maia Queiroz Lima ${ }^{5}$, Telton Pedro Anselmo Ramos ${ }^{5,6}$ \&

Jorge Iván Sánchez-Botero ${ }^{2 *}$

${ }^{I}$ Instituto Nacional de Pesquisas da Amazônia, Programa de Pós-Graduação em Ecologia, Av. André Araújo, 2936, Manaus, AM, Brazil

${ }^{2}$ Universidade Federal do Ceará, Departamento de Biologia, Laboratório de Ecologia Aquática, Fortaleza, CE, Brazil

${ }^{3}$ Universidade Federal do Paraná, Centro Politécnico, Curitiba, PR, Brazil

${ }^{4}$ Universidade Federal do Ceará, LABOMAR: Instituto de Ciências do Mar, Fortaleza, CE, Brazil

${ }^{5}$ Universidade Federal do Rio Grande do Norte, Departamento de Botânica e Zoologia, Avenida Senador Salgado Filho, 3000, Lagoa Nova, Natal, RN, Brazil

${ }^{6}$ Universidade Federal da Paraíba, Centro de Ciências Exatas e da Natureza, Departamento de Sistemática e Ecologia, Campus I, João Pessoa, PB, Brazil

*Corresponding author: Jorge Iván Sánchez-Botero, e-mail: jisbar.ufc@gmail.com

RODRIGUES-FILHO, C.A.S., GURGEL-LOURENÇO, R.C., BEZERRA, L.A.V., SOUSA, W.A., GARCEZ, D.S., LIMA, S.M.Q., RAMOS, T.P.A., SÁNCHEZ-BOTERO, J.I. Ichthyofauna of the humid forest enclaves in the tablelands of Ibiapaba and Araripe, Northeastern Brazil. Biota Neotropica. 16(4): e20160273. http://dx.doi. org/10.1590/1676-0611-BN-2016-0273

\begin{abstract}
Humid highland forest enclaves are remnants of Atlantic Forest found in tablelands within the Caatinga biome (Northeastern Brazil), which emerged during interglacial periods in the Pleistocene. These ecosystems have a highly diverse and endemic fish fauna. Most earlier surveys have focused on the tableland of Borborema (Pernambuco and Paraíba States). In this study we surveyed the fish fauna of the humid forest enclaves in the tablelands of Ibiapaba and Araripe, based on samples collected in the rainy season (March and April) between 2009 and 2014. The 45 sampling points covered rivers, streams and reservoirs in five river basins belonging to three ecoregions. The species were listed according to drainage divide, and endemism was determined for each ecoregion and for the Caatinga. Our area was more species-rich $(n=59)$ than Borborema $(n=27)$. The samples included five introduced species and 29 species endemic to the Caatinga (49.1\% of the sampled species). The distribution of Parotocinclus haroldoi was expanded to the Mid-Northeastern Caatinga ecoregion (Timonha river basin, Ceará State). Our study intends to make a significant contribution to current knowledge of the ichthyofauna in humid highland forest enclaves of semiarid Northeastern Brazil, identified as a priority in the conservation of the biodiversity in the Caatinga.
\end{abstract}

Keywords: Neotropical Region, Endemism, Conservation, Fishes of the Caatinga.

\section{Ictiofauna dos enclaves de floresta úmida nos planaltos da Ibiapaba e do Araripe, Nordeste do Brasil}

\footnotetext{
Resumo: Florestas úmidas são enclaves de Mata Atlântica em regiões elevadas inseridas no bioma Caatinga que surgiram no Pleistoceno em eventos interglaciais. Esses ecossistemas abrigam alta diversidade de espécies para diversos táxons e elevadas taxas de endemismo. A maioria dos levantamentos de peixes nesses ecossistemas foi realizada no Planalto da Borborema, nos Estados de Pernambuco e Paraíba. Dessa forma, visando conhecer a ictiofauna de dois enclaves de florestas úmidas no nordeste brasileiro, foram feitas coletas no período chuvoso (Março e Abril) entre 2009 e 2014 no Planalto da Ibiapaba e na Chapada do Araripe. Foram amostrados 45 pontos em rios, riachos e reservatórios em cinco bacias hidrográficas e três ecorregiões. A lista de espécies por drenagem e o endemismo foi definido para cada ecorregião e para a Caatinga. A riqueza de peixes (59 espécies) foi superior quando comparada a dos enclaves da Borborema (27), com cinco espécies introduzidas e 29 endêmicas para a Caatinga, o que representa 49,1\% das espécies capturadas. Destaca-se a expansão de ocorrência de Parotocinclus haroldoi para a ecorregião do Nordeste Médio-Oriental, na
} 
microbacia do rio Timonha, no Estado do Ceará. Este estudo pretende contribuir com informações inéditas para o conhecimento ictiofaunístico dos ecossistemas aquáticos dos brejos de alitude do semiárido brasileiro, apontadas como prioritárias para conservação da biodiversidade da Caatinga.

Palavras-chave: Região Neotropical, Endemismo, Conservação, Peixes da Caatinga.

\section{Introduction}

The Brazilian territory may be divided into six large morphoclimatic zones, each with its own climatic, pedological, hydrological and phytogeographic characteristics (Ab'Sáber 2003). One of these, the Caatinga, a predominantly Northeast Brazilian biome, is characterized by a semiarid climate with a relatively short rainy season (four months) of sporadic and unevenly distributed rainfalls. Due to the paucity of rain (240-800 mm annually) and high evaporation rate (Tabarelli \& Santos 2004), most aquatic systems are intermittent. This probably results in a smaller fish diversity than that observed in other tropical aquatic systems (Medeiros \& Maltchik 2001). However, the Caatinga features minor contrasting areas, such as humid highland forest enclaves locally referred to as 'brejos de altitude' (Ab'Sáber 2003). Acting as drainage divides between the main hydrographic ecoregions of Northeastern Brazil (Maranhão-Piauí, Mid-Northeastern Caatinga, São Francisco and Northeastern Atlantic Forest), these highland enclaves receive more rain than the surrounding Caatinga due to orographic rainfalls, favoring the maintenance of aquatic systems throughout the year. Moreover, the expansion and posterior recession of the Atlantic and Amazon forest in Northeastern Brazil due to climate changes in the Pleistocene (Andrade-Lima 1982, Santos et al. 2007) resulted in the emergence of unique ecosystems with high levels of diversity and endemism, which serve as refuges for regional fauna and flora (Andrade-Lima 1982).

Unfortunately, little is known about the aquatic biota of the Brazilian semiarid region and how fish assemblages and richness are impacted by environmental degradation and the introduction of exotic species (Santos et al. 2011, Botero et al. 2014). In addition, efforts at freshwater fish conservation have been feeble at best (Lévêque et al. 2008, Abell et al. 2008). Fish fauna surveys not only help define biogeographic regions, but also provide important subsidies for environmental policy decisions (especially with regard to the establishment and effectiveness of conservation areas) and serve as an important first step for research in related fields (Albert et al. 2011).

Rosa \& Groth (2004) published the first comprehensive study on fish assemblages in humid highland forest enclaves. The study covered the Borborema tableland which drains into the Paraíba do Norte and Mamanguape river basins (Paraíba State) and the Ipojuca river basin (Pernambuco State) located in the eastern part of the Mid-Northeastern Caatinga ecoregion. Other initiatives, such as the PROBIO Project for Conservation and Sustainable Use of Brazilian Biological Diversity (Projeto de Conservação e Utilização Sustentável da Diversidade Biológica Brasileira) and the PPBio Program for Research in Biodiversity (Programa de Pesquisa em Biodiversidade), are helping expand current knowledge on fish diversity in the region. In addition, Ramos et al. (2014) recently evaluated the richness of the ichthyofauna of the Parnaíba river basin (a semiarid region shared by Ceará, Piauí and Maranhão States) and identified 146 species of freshwater fishes, $54(36.9 \%)$ of which were endemic to the basin. Their survey added significantly to the list of 95 species of Albert et al. (2011) for the Maranhão-Piauí ecoregion, highlighting the need for additional inventories. Nevertheless, many gaps remain in the knowledge of the ichthyofauna of the São Francisco and Mid-Northeastern Caatinga ecoregions, especially with regard to humid forest enclaves (Rosa \& Groth 2004, Langeani et al. 2009). Thus, the purpose of this study was to provide a list of fish species from forest enclaves and streams in the tablelands of Ibiapaba (between
Piauí and Ceará States) and Araripe (between Ceará and Pernambuco States) which drain into the Parnaíba, Coreaú, Timonha, Jaguaribe and São Francisco river basins.

\section{Materials and methods}

\section{Study area}

The surveys were conducted in humid forest enclaves in two tablelands, Ibiapaba and Araripe, which act as drainage divides between the Maranhão-Piauí, São Francisco and Mid-Northeastern Caatinga ecoregions (Rosa et al. 2003, Albert et al. 2011) (Figure 1). The two tablelands make up the Araripe-Ibiapaba complex, which extends along the confines from Northwestern Ceará to Northeastern Piauí and from Southern Ceará to Central Piauí. The complex displays great altitude variations between the highlands and the central lowlands (depressão sertaneja). In the highlands, soils (latosols) tend to be deep, sandy and poor, and surface water is scarce. In Ibiapaba, water infiltrating the soil drains mainly towards Piauí State. In Araripe, water drains towards Ceará State.

Araripe and Ibiapaba have a maximum elevation of 900-1,000 m (Claudino-Sales \& Lira 2011), average rainfalls of 1,000 $\mathrm{mm}$ in the rainy season (Sá et al. 2004) and perennial or intermittent streams. The humid forests of Ibiapaba drain westward into the Parnaíba basin (Maranhão-Piauí) in Piauí and eastward into the Coreaú basin (Mid-Northeastern Caatinga) in Ceará, whereas Araripe drains northward into the Jaguaribe basin (Mid-Northeastern Caatinga) in Ceará and southward into the São Francisco basin (Rosa et al. 2003) in Pernambuco State (Figure 1).

The eastern slope of the Ibiapaba tableland intercepts the humid coastal wind producing orographic rainfalls and making this region the wettest (mean annual rainfall: $\sim 1000 \mathrm{~mm}$ ) and coolest (mean annual temperature: $22-26^{\circ} \mathrm{C}$ ) in Ceará. On the other hand, Araripe has $8,000 \mathrm{~km}^{2}$ of highland forest and elevations up to $1,000 \mathrm{~m}$ (Köppen type BSh'), with mean annual temperatures around $25^{\circ} \mathrm{C}$ (MME 1996, SRH 2005). These enclaves were previously considered a remnant of the Atlantic Forest (due to the geographic proximity), but recent studies have shown the flora to be more akin to that of the Amazon Forest (Santos et al. 2007).

\section{Sampling}

Fish were sampled in the rainy season (March-April) during six surveys from 2009 to 2014, covering 45 sampling points-24 in Araripe and 21 in Ibiapaba (Table 1, Figure 2). The sampling sites included first to third-order rivers $(n=9)$, streams $(n=27)$ and small reservoirs $(n=9)$ $\left(100-1,000 \mathrm{~m}^{2}\right)$. The sampling points in Araripe were located within the Jaguaribe river basin $(n=7)$ and the São Francisco river basin $(n=17)$, while the sampling points in Ibiapaba were located within the Coreaú river basin $(\mathrm{n}=12)$, the Parnaíba river basin $(\mathrm{n}=8)$ and the Timonha river basin $(\mathrm{n}=1)$ (Table 1). Each sampling site was georeferenced using a portable GPS and later plotted on a map to avoid errors of attribution in areas near drainage divides. Using the definition of Rosa et al. (2003), only species restricted to a single ecoregion (Albert et al. 2011) were considered endemic.

Seventeen of the 45 sampling points were located inside conservation units. Thus, seven of the sampling points in the Jaguaribe river basin and one in the São Francisco river basin were located inside the Chapada do Araripe Environmental Protection Area (EPA) (1,063,000 ha). In Ibiapaba, one sampling point in the Timonha river basin was located in the Serra da Ibiapaba EPA 


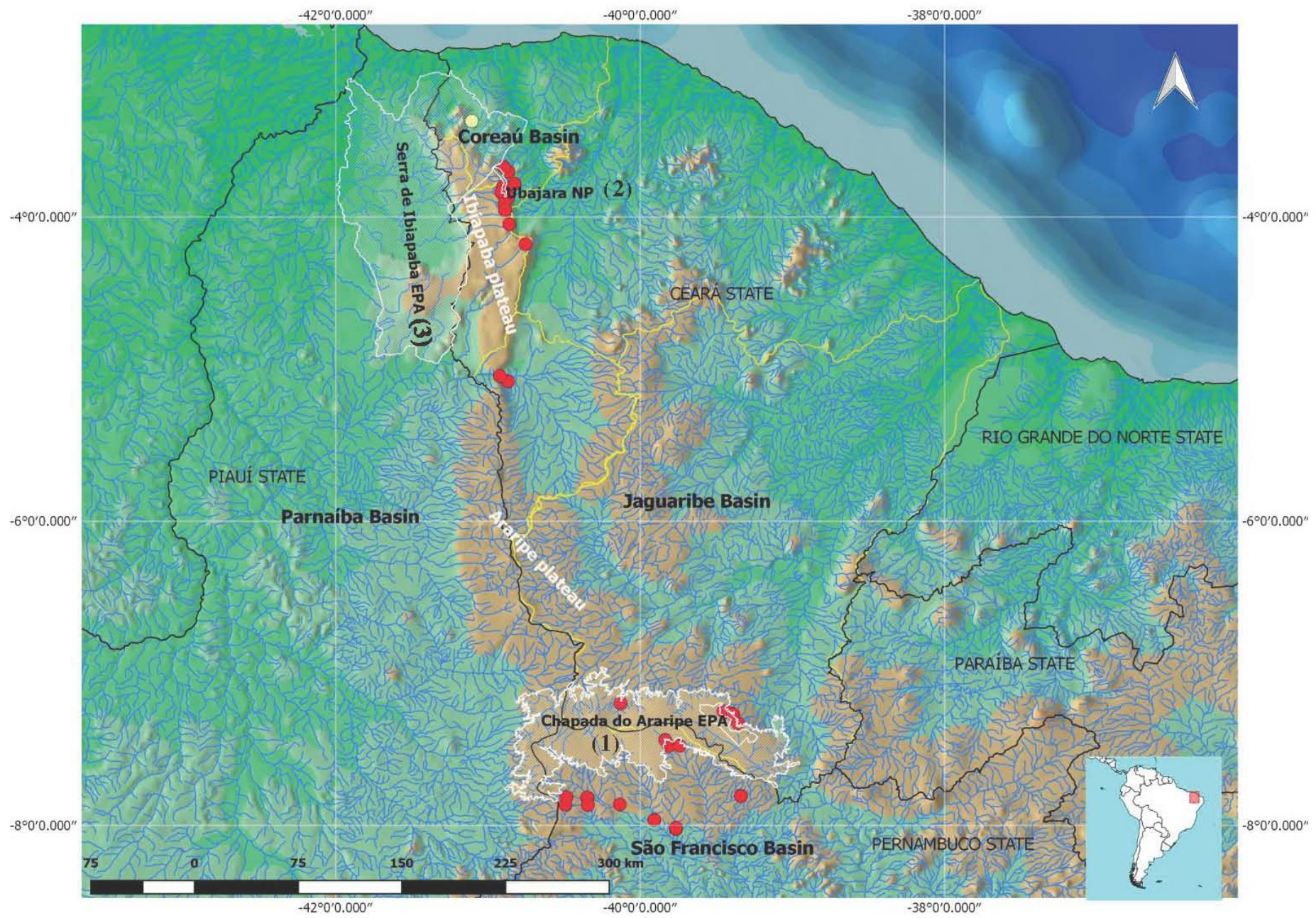

Figure 1. Study area and sampling points in Northeastern Brazil. The sampling sites are represented by red dots (the Coreaú, Jaguaribe, Parnaíba and São Francisco basins) and yellow (Timonha river basin). The tablelands are shown in brown. Conservation units: (1) Chapada do Araripe EPA; (2) Ubajara National Park (NP); (3) Serra da Ibiapaba EPA.

(1,592,550 ha) and eight sampling points in the Coreaú river basin were located in the Ubajara National Park (NP) (6,271 ha).

The fish were captured with casting nets $\left(5.3 \mathrm{~m}^{2} ; 14 \mathrm{~mm}\right.$ between opposing knots), sieves $\left(0.7 \mathrm{~m}^{2} ; 1 \mathrm{~mm}\right.$ between opposing knots) and seines $\left(1.3 \mathrm{~m}^{2} ; 2 \mathrm{~mm}\right.$ between opposing knots) in all accessible habitats (aquatic macrophytes, ponds, pools, rapids and waterfalls) with a fishing effort of four man-hours, under licenses (\#26174-2,\#32656 and \#32921-4) issued by the Ministry of the Environment (MMA) and the Chico Mendes Institute of Biodiversity Conservation (ICMBio). The ichthyological material was deposited in the fish collection of the Universidade Federal do Rio Grande do Norte (UFRN) and the Universidade Federal da Paraíba (UFPB). Vouchers for each species are included in the appendix.

\section{Results and discussion}

\section{Ichthyofauna and endemism}

Fifty-nine fish species, belonging to 38 genera, 16 families and five orders, were identified in our samples from the two tablelands (Table 2). This is more than twice the number of species (27) reported by Rosa \& Groth (2004) for nine humid forest enclaves in the tableland of Borborema (Mid-Northeastern Caatinga ecoregion). As pointed out by the authors, the low diversity observed in that study, when compared to that of larger regional river basins, may be the result of sampling or logistic limitations. We do not intend to have fully overcome the difficulties described over a decade ago (for example, not all stretches of the sampled tributaries were accessible due to steep banks, dense riparian vegetation and fast-flowing waters), but with the help of new research teams from several universities in semiarid Northeastern Brazil and taxonomic advances, we believe to have produced a comprehensive and fairly accurate list of the fish species in these ecosystems.

Current knowledge of fish diversity and endemicity in the Caatinga is mainly based on surveys conducted in the largest basins of Northeastern Brazil (Rosa et al. 2003, Rosa \& Groth 2004). Miranda-Ribeiro (1937) were the first to describe the fish fauna of humid highland forests in the region (Granjeiro river basin, Araripe). Nijssen and Isbrüker (1976) identified species of Aspidoras in highland streams in Northeastern Brazil (mostly in Ceará). More recently, Rosa \& Groth (2004) published a seminal study on humid highland forest fish fauna, with emphasis on the question of endemicity and the urgent need for conservation.

None of the species registered in this study (37 in Araripe and 33 in Ibiapaba) is on Brazil's official list of endangered fishes and aquatic invertebrates (MMA, 2014). When introduced species were excluded ( $\mathrm{n}=5)$ from the analysis, the two tablelands had nine species in common, and 
Rodrigues-Filho, C.A.S. et al.

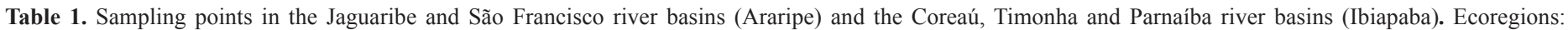

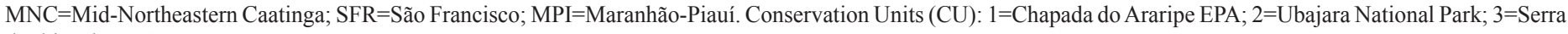
da Ibiapaba EPA.

\begin{tabular}{|c|c|c|c|c|c|}
\hline Tableland & Basin & Ecoregion & Habitat & $\mathbf{C U}$ & Geographic coordinates \\
\hline Araripe & Jaguaribe & $\mathrm{MNC}$ & Stream & 1 & $7^{\circ} 16^{\prime} 01^{\prime \prime S} 39^{\circ} 23^{\prime} 36^{\prime \prime} \mathrm{W}$ \\
\hline Araripe & Jaguaribe & $\mathrm{MNC}$ & Stream & 1 & $7^{\circ} 14^{\prime} 56^{\prime \prime S} 39^{\circ} 27^{\prime} 12^{\prime \prime} \mathrm{W}$ \\
\hline Araripe & Jaguaribe & $\mathrm{MNC}$ & Stream & 1 & $7^{\circ} 16^{\prime} 12^{\prime \prime S} 39^{\circ} 23^{\prime} 20^{\prime \prime} \mathrm{W}$ \\
\hline Araripe & Jaguaribe & $\mathrm{MNC}$ & Reservoir & 1 & $7^{\circ} 11^{\prime} 38^{\prime \prime} \mathrm{S} 40^{\circ} 07^{\prime} 41^{\prime \prime} \mathrm{W}$ \\
\hline Araripe & Jaguaribe & $\mathrm{MNC}$ & Reservoir & 1 & $7^{\circ} 11^{\prime} 58^{\prime \prime} \mathrm{S} 40^{\circ} 07^{\prime} 45^{\prime \prime} \mathrm{W}$ \\
\hline Araripe & Jaguaribe & $\mathrm{MNC}$ & River & 1 & $7^{\circ} 15^{\prime} 18^{\prime \prime} \mathrm{S} 39^{\circ} 25^{\prime} 58^{\prime \prime} \mathrm{W}$ \\
\hline Araripe & Jaguaribe & $\mathrm{MNC}$ & River & 1 & $7^{\circ} 19^{\prime} 41^{\prime \prime S} 39^{\circ} 21^{\prime} 26^{\prime \prime} \mathrm{W}$ \\
\hline Araripe & São Francisco & SFR & Stream & - & $7^{\circ} 11^{\prime} 58^{\prime \prime} \mathrm{S} 40^{\circ} 07^{\prime} 45^{\prime \prime} \mathrm{W}$ \\
\hline Araripe & São Francisco & SFR & Stream & - & $7^{\circ} 48^{\prime} 19^{\prime \prime} \mathrm{S} 39^{\circ} 20^{\prime} 19^{\prime \prime} \mathrm{W}$ \\
\hline Araripe & São Francisco & SFR & Stream & - & $7^{\circ} 28^{\prime} 34^{\prime \prime} \mathrm{S} 39^{\circ} 48^{\prime} 45^{\prime \prime} \mathrm{W}$ \\
\hline Araripe & São Francisco & SFR & Stream & - & $7^{\circ} 28^{\prime} 34^{\prime \prime} \mathrm{S} 39^{\circ} 48^{\prime} 45^{\prime \prime} \mathrm{W}$ \\
\hline Araripe & São Francisco & SFR & Stream & - & $7^{\circ} 26^{\prime} 11^{\prime \prime S} 39^{\circ} 50^{\prime} 10^{\prime \prime} \mathrm{W}$ \\
\hline Araripe & São Francisco & SFR & Stream & - & $7^{\circ} 49^{\prime} 06 ” \mathrm{~S} 40^{\circ} 20^{\prime} 37^{\prime \prime} \mathrm{W}$ \\
\hline Araripe & São Francisco & SFR & Stream & - & $7^{\circ} 51^{\prime} 40^{\prime \prime S} 40^{\circ} 08^{\prime} 02^{\prime \prime} \mathrm{W}$ \\
\hline Araripe & São Francisco & SFR & Stream & - & $8^{\circ} 00^{\prime} 52^{\prime \prime} \mathrm{S} 39^{\circ} 45^{\prime} 54^{\prime \prime} \mathrm{W}$ \\
\hline Araripe & São Francisco & SFR & Stream & - & $8^{\circ} 01^{\prime} 25^{\prime \prime} \mathrm{S} 39^{\circ} 45^{\prime} 54^{\prime \prime} \mathrm{W}$ \\
\hline Araripe & São Francisco & SFR & Stream & - & $7^{\circ} 48^{\prime} 58^{\prime \prime} \mathrm{S} 40^{\circ} 20^{\prime} 56^{\prime \prime} \mathrm{W}$ \\
\hline Araripe & São Francisco & SFR & Reservoir & 1 & $7^{\circ} 26^{\prime} 30^{\prime \prime} \mathrm{S} 39^{\circ} 50^{\prime} 01^{\prime \prime} \mathrm{W}$ \\
\hline Araripe & São Francisco & SFR & Reservoir & - & $7^{\circ} 51^{\prime} 49$ 'S 40²9'29”W \\
\hline Araripe & São Francisco & SFR & Reservoir & - & $7^{\circ} 49^{\prime} 06 ” \mathrm{~S} 40^{\circ} 20^{\prime} 37^{\prime \prime} \mathrm{W}$ \\
\hline Araripe & São Francisco & SFR & Reservoir & - & $7^{\circ} 48^{\prime} 50^{\prime \prime} \mathrm{S} 40^{\circ} 29^{\prime} 03^{\prime \prime} \mathrm{W}$ \\
\hline Araripe & São Francisco & SFR & Reservoir & - & $7^{\circ} 51^{\prime} 59$ 's $40^{\circ} 20^{\prime} 37^{\prime \prime} \mathrm{W}$ \\
\hline Araripe & São Francisco & SFR & Reservoir & - & 7०57’44”S 39॰54’27’W \\
\hline Araripe & São Francisco & SFR & River & - & $7^{\circ} 48^{\prime} 58^{\prime \prime} \mathrm{S} 40^{\circ} 20^{\prime} 56^{\prime \prime} \mathrm{W}$ \\
\hline Ibiapaba & Coreaú & $\mathrm{MNC}$ & Stream & 2 & $3^{\circ} 50^{\prime} 00^{\prime \prime} \mathrm{S} 40^{\circ} 54^{\prime} 00^{\prime \prime} \mathrm{W}$ \\
\hline Ibiapaba & Coreaú & $\mathrm{MNC}$ & Stream & 2 & $3^{\circ} 50^{\prime} 22^{\prime \prime S} 40^{\circ} 54^{\prime} 29^{\prime \prime} \mathrm{W}$ \\
\hline Ibiapaba & Coreaú & $\mathrm{MNC}$ & Stream & 2 & $3^{\circ} 50^{\prime} 07^{\prime \prime} \mathrm{S} 40^{\circ} 54^{\prime} 03^{\prime \prime} \mathrm{W}$ \\
\hline Ibiapaba & Coreaú & $\mathrm{MNC}$ & Stream & 2 & $3^{\circ} 50^{\prime} 00^{\prime \prime} \mathrm{S} 40^{\circ} 53^{\prime} 22^{\prime \prime} \mathrm{W}$ \\
\hline Ibiapaba & Coreaú & $\mathrm{MNC}$ & Stream & 2 & $3^{\circ} 49^{\prime} 48^{\prime \prime} \mathrm{S} 40^{\circ} 54^{\prime} 02^{\prime \prime} \mathrm{W}$ \\
\hline Ibiapaba & Coreaú & $\mathrm{MNC}$ & Stream & 2 & $3^{\circ} 48^{\prime} 05^{\prime \prime} \mathrm{S} 40^{\circ} 54^{\prime} 06^{\prime \prime} \mathrm{W}$ \\
\hline Ibiapaba & Coreaú & $\mathrm{MNC}$ & Stream & 2 & $3^{\circ} 50^{\prime} 16^{\prime \prime} \mathrm{S} 40^{\circ} 54^{\prime} 46^{\prime \prime} \mathrm{W}$ \\
\hline Ibiapaba & Coreaú & $\mathrm{MNC}$ & Stream & - & $3^{\circ} 52^{\prime} 06^{\prime \prime} \mathrm{S} 40^{\circ} 51^{\prime} 59^{\prime \prime} \mathrm{W}$ \\
\hline Ibiapaba & Coreaú & $\mathrm{MNC}$ & Stream & - & $3^{\circ} 40^{\prime} 17^{\prime \prime} \mathrm{S} 40^{\circ} 53^{\prime} 44^{\prime \prime} \mathrm{W}$ \\
\hline Ibiapaba & Coreaú & $\mathrm{MNC}$ & Stream & - & $3^{\circ} 42^{\prime} 09^{\prime \prime} \mathrm{S} 40^{\circ} 51^{\prime} 53^{\prime \prime} \mathrm{W}$ \\
\hline Ibiapaba & Coreaú & $\mathrm{MNC}$ & River & 2 & $3^{\circ} 49^{\prime} 32^{\prime \prime} \mathrm{S} 40^{\circ} 53^{\prime} 30^{\prime \prime} \mathrm{W}$ \\
\hline Ibiapaba & Coreaú & $\mathrm{MNC}$ & Reservoir & - & $3^{\circ} 46^{\prime} 51^{\prime \prime} \mathrm{S} 40^{\circ} 49^{\prime} 32^{\prime \prime} \mathrm{W}$ \\
\hline Ibiapaba & Timonha & $\mathrm{MNC}$ & Stream & 3 & $3^{\circ} 22^{\prime} 07^{\prime \prime} \mathrm{S} 41^{\circ} 06^{\prime} 23^{\prime \prime} \mathrm{W}$ \\
\hline Ibiapaba & Parnaíba & MPI & Stream & - & $5^{\circ} 19^{\prime} 13^{\prime \prime} \mathrm{S} 40^{\circ} 26^{\prime} 43^{\prime \prime} \mathrm{W}$ \\
\hline Ibiapaba & Parnaíba & MPI & Stream & - & $5^{\circ} 04^{\prime} 49^{\prime} \mathrm{S} 40^{\circ} 52^{\prime} 01^{\prime \prime} \mathrm{W}$ \\
\hline Ibiapaba & Parnaíba & MPI & Stream & - & $5^{\circ} 08^{\prime} 09^{\prime \prime} \mathrm{S} 40^{\circ} 46^{\prime} 03^{\prime \prime} \mathrm{W}$ \\
\hline Ibiapaba & Parnaíba & MPI & River & - & $5^{\circ} 02^{\prime} 42^{\prime \prime} \mathrm{S} 40^{\circ} 55^{\prime} 16^{\prime \prime} \mathrm{W}$ \\
\hline Ibiapaba & Parnaíba & MPI & River & - & $3^{\circ} 54^{\prime} 47^{\prime \prime} \mathrm{S} 40^{\circ} 53^{\prime} 25^{\prime \prime} \mathrm{W}$ \\
\hline Ibiapaba & Parnaíba & MPI & River & - & $4^{\circ} 02^{\prime} 48^{\prime \prime} \mathrm{S} 40^{\circ} 51^{\prime} 37^{\prime \prime} \mathrm{W}$ \\
\hline Ibiapaba & Parnaíba & MPI & River & - & $3^{\circ} 57^{\prime} 11^{\prime \prime} \mathrm{S} 40^{\circ} 53^{\prime} 13^{\prime \prime} \mathrm{W}$ \\
\hline Ibiapaba & Parnaíba & MPI & River & - & $4^{\circ} 10^{\prime} 41^{\prime \prime S} 40^{\circ} 45^{\prime} 09^{\prime \prime} \mathrm{W}$ \\
\hline
\end{tabular}

29 species (53.7\%) endemic to the Caatinga (Araripe $\mathrm{n}=18,56.2 \%$; Ibiapaba $\mathrm{n}=15 ; 51.7 \%$ ) (Table 2) (Rosa et al. 2003). When only the endemic species were considered, endemism was greater in Araripe (28.1\%) than in Ibiapaba (20.0\%) (Figure 3).

The most species-rich ecoregion was Mid-Northeastern Caatinga $(n=29)$, followed by Maranhão-Piauí $(n=26)$ and São Francisco $(n=24)$. The ecoregions did not differ regard the number of endemic species: six $(24.0 \%)$ in Mid-Northeastern Caatinga, four (20.0\%) in São Francisco and five (19.0\%) in Maranhão-Piauí (Figure 3). In view of the fact that only a small part of each ecoregion was sampled, the observed levels of endemism may be considered high.

In addition to harboring a large number of fishes of the Caatinga ( $\sim 56 \%$ endemicity), humid forest enclaves play a crucial role in the maintenance and conservation of the regional ichthyofauna (Rosa \& Groth 2004). In this study, the most species-rich river basin was Parnaíba $(n=26)$, followed by São Francisco $(n=24)$, Jaguaribe $(n=20)$, Coreaú $(n=16)$ and Timonha 


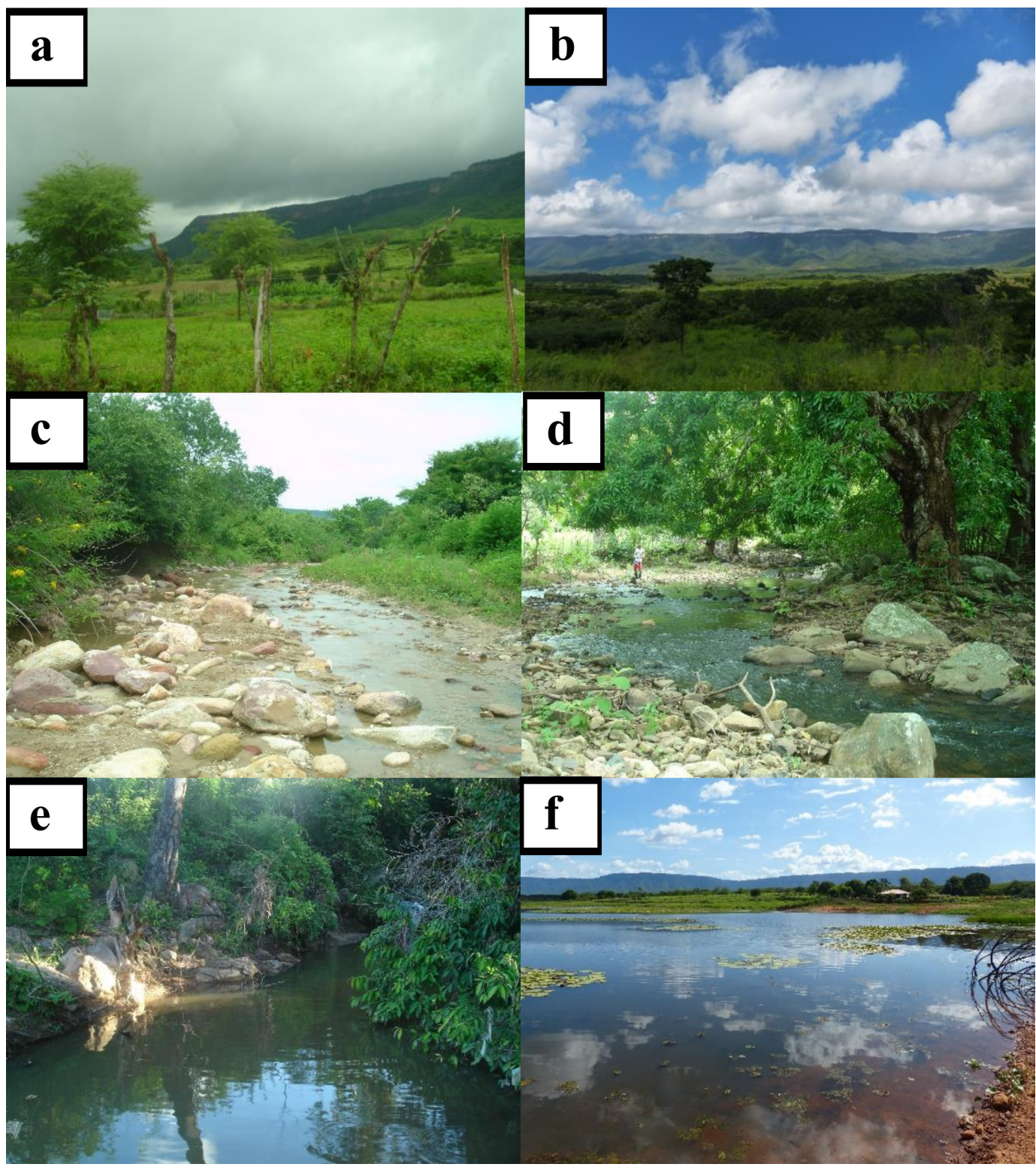

Figure 2. The tablelands of Araripe (a) and Ibiapaba (b), with some sample points: (c) Salamanca river, (d) Tamundé stream, (e) Passagem stream, and (f) Barreira reservoir.

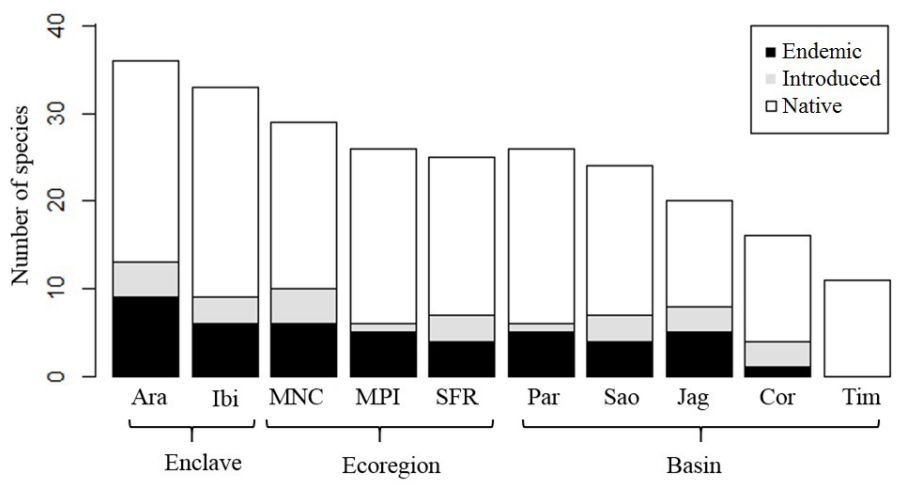

Figure 3. Number of fish species registered for the tablelands of Ibiapaba (Ibi) and Araripe (Ara), for the ecoregions Mid-Northeastern Caatinga (MNC), Maranhão-Piauí (MPI) and São Francisco (SFR), and for the river basins of Parnaíba (Par), São Francisco (Sao), Jaguaribe (Jag), Coreaú (Cor) and Timonha (Tim). The number of endemic species was determined for each ecoregion. $(n=11)$ (Table 2). When analyzed according to ecoregion, Jaguaribe and Parnaíba both yielded five endemic species, followed by São Francisco $(\mathrm{n}=4)$, Coreaú $(\mathrm{n}=1)$ and Timonha $(\mathrm{n}=0)$ (Figure 3).

The introduced species registered in this study (Cichla sp., Coptodon rendalli, Oreochromis niloticus, Poecilia reticulata and Xiphophorus helleri) (Table 2 ) have a potentially negative impact on native fish diversity mainly through taxonomic homogenization of assemblages and species extinction (Vitule et al. 2009, Villéger et al. 2014). The introduced species (except the last one) were also observed by Rosa \& Groth (2004) in the humid forest enclaves of Borborema. The introduction of species into humid forest enclaves in Northeastern Brazil is of particular concern in view of the high level of endemism, the scarcity of information and related anthropic impacts (e.g., removal of riparian vegetation, building of reservoirs and use of agricultural pesticides) (Rosa \& Groth 2004). For example, due to the predominance of small fishes in the local assemblage, the abundance and wide distribution of $P$. reticulata may lead to the competitive exclusion of native species, especially poeciliids (Rosa \& Groth 2004), as Poecilia sp., observed in the Timonha river basin. 


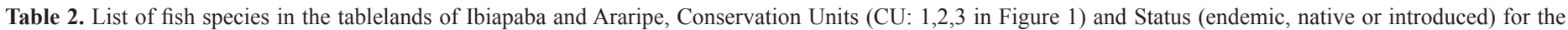

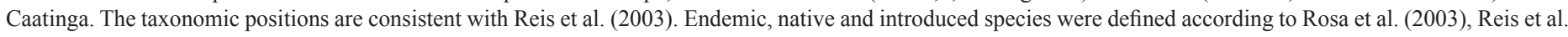
(2003) and Ramos et al. (2014).

\begin{tabular}{|c|c|c|c|c|c|c|c|}
\hline \multirow[b]{2}{*}{ Taxon } & \multicolumn{3}{|c|}{ Ibiapaba } & \multicolumn{2}{|c|}{ Araripe } & \multirow[b]{2}{*}{$\mathbf{C U}$} & \multirow[b]{2}{*}{$\begin{array}{c}\text { Status } \\
\text { Caatinga }\end{array}$} \\
\hline & $\begin{array}{c}\text { Parnaíba } \\
\text { (MPI) }\end{array}$ & $\begin{array}{l}\text { Coreaú } \\
\text { (MNC) }\end{array}$ & $\begin{array}{c}\text { Timonha } \\
\text { (MNC) }\end{array}$ & $\begin{array}{c}\text { Jaguaribe } \\
\text { (MNC) }\end{array}$ & $\begin{array}{c}\text { São } \\
\text { Francisco } \\
\text { (SFR) }\end{array}$ & & \\
\hline \multicolumn{8}{|l|}{ CHARACIFORMES } \\
\hline \multicolumn{8}{|l|}{ Parodontidae } \\
\hline Apareiodon hasemani ${ }^{1}$ Eigenmann, 1916 & & & & & $\mathrm{X}^{(\mathrm{E})}$ & & Endemic \\
\hline \multicolumn{8}{|l|}{ Curimatidae } \\
\hline Curimatella lepidura ${ }^{2}$ (Eigenmann \& Eigenmann, 1889) & & & & & $\mathrm{X}$ & & Endemic \\
\hline Steindachnerina elegans ${ }^{3}$ (Steindachner, 1875) & & & & & $\mathrm{X}$ & & Native \\
\hline Steindachnerina notonota ${ }^{4}$ (Miranda Ribeiro, 1937) & $\mathrm{X}$ & $\mathrm{X}$ & $X$ & $\mathrm{X}$ & & 1,3 & Endemic \\
\hline \multicolumn{8}{|l|}{ Prochilodontidae } \\
\hline Prochilodus brevis ${ }^{5}$ Steindachner, 1875 & & & & & $\mathrm{X}$ & & Endemic \\
\hline Prochilodus lacustris ${ }^{6}$ Steindachner, 1907 & $\mathrm{X}^{(\mathrm{E})}$ & & & & & & Endemic \\
\hline \multicolumn{8}{|l|}{ Anostomidae } \\
\hline Leporinus piau ${ }^{7}$ Fowler, 1941 & $\mathrm{X}$ & & & & & & Endemic \\
\hline \multicolumn{8}{|l|}{ Crenuchidae } \\
\hline Characidium bimaculatum $^{8}$ Fowler, 1941 & $\mathrm{X}$ & $\mathrm{X}$ & $X$ & $X$ & $\mathrm{X}$ & $1,2,3$ & Endemic \\
\hline \multicolumn{8}{|l|}{ Characidae } \\
\hline Astyanax aff. bimaculatus ${ }^{9}$ (Linnaeus, 1758) & $\mathrm{X}$ & $\mathrm{X}$ & $X$ & $\mathrm{X}$ & & $1,2,3$ & Native \\
\hline Astyanax aff. fasciatus ${ }^{10}$ (Cuvier, 1819) & $\mathrm{X}$ & $X$ & $\mathrm{X}$ & $\mathrm{X}$ & & 1,3 & Native \\
\hline Astyanax lacustris ${ }^{11}$ (Lütken, 1875) & & & & & $\mathrm{X}$ & & Native \\
\hline Astyanax rivularis ${ }^{12}$ (Lütken, 1875) & & & & & $\mathrm{X}$ & & Native \\
\hline Compsura heterura ${ }^{13}$ Eigenmann, 1915 & $\mathrm{X}$ & & & & & & Endemic \\
\hline Creagrutus sp. ${ }^{14}$ & $\mathrm{X}$ & & & & & & Native \\
\hline Hemigrammus marginatus ${ }^{15}$ Ellis, 1911 & $\mathrm{X}$ & & & & & & Native \\
\hline Hemigrammus rodwayi ${ }^{16}$ Durbin, 1909 & $\mathrm{X}$ & & & & & & Native \\
\hline Knodus victoriae ${ }^{17}$ (Steindachner, 1907) & $\mathrm{X}^{(\mathrm{E})}$ & & & & & & Endemic \\
\hline Moenkhausia costae ${ }^{18}$ (Steindachner, 1907) & & & & & $\mathrm{X}$ & & Endemic \\
\hline Orthospinus franciscensis ${ }^{19}$ (Eigenmann, 1914) & & & & & $X^{(E)}$ & & Endemic \\
\hline Phenacogaster calverti ${ }^{20}$ (Fowler, 1941) & $\mathrm{X}$ & $\mathrm{X}$ & & & & & Endemic \\
\hline Psellogrammus kennedyi ${ }^{21}$ (Eigenmann, 1903) & & & & & $X$ & & Native \\
\hline Serrapinnus heterodon 22 (Eigenmann, 1915) & $\mathrm{X}$ & & $\mathrm{X}$ & $\mathrm{X}$ & $\mathrm{X}$ & 1,3 & Native \\
\hline Serrapinnus piaba ${ }^{23}$ (Lütken, 1875) & $\mathrm{X}$ & $X$ & $X$ & & & 3 & Native \\
\hline Tetragonopterus chalceus ${ }^{24}$ Spix \& Agassiz, 1829 & & & & & $\mathrm{X}$ & & Native \\
\hline \multicolumn{8}{|l|}{ Serrasalmidae } \\
\hline Pygocentrus nattereri ${ }^{25}$ Kner, 1858 & $\mathrm{X}$ & & & & & & Native \\
\hline \multicolumn{8}{|l|}{ Erythrinidae } \\
\hline Hoplerythrinus unitaeniatus ${ }^{26}$ (Spix \& Agassiz, 1829) & $\mathrm{X}$ & & & & & & \\
\hline Hoplias malabaricus ${ }^{27}$ (Bloch, 1794) & $\mathrm{X}$ & $X$ & & $X$ & $X$ & 1,2 & Native \\
\hline Triportheidae & & & & & & & Native \\
\hline Triportheus guentheri ${ }^{28}$ (Garman, 1890) & & & & & $X^{(E)}$ & & Endemic \\
\hline \multicolumn{8}{|l|}{ SILURIFORMES } \\
\hline \multicolumn{8}{|l|}{ Callichthyidae } \\
\hline Aspidoras menezesi ${ }^{29}$ Nijssen \& Isbrücker, 1976 & & & & $\mathrm{X}^{(\mathrm{E})}$ & & 1 & Endemic \\
\hline Aspidoras raimundi $^{30}$ (Steindachner, 1907) & $X^{(E)}$ & & & & & & Endemic \\
\hline Aspidoras rochai ${ }^{31}$ Ihering, 1907 & & & & & $\mathrm{X}$ & & Endemic \\
\hline Aspidoras sp. ${ }^{32}$ & & & & & $\mathrm{X}$ & & Native \\
\hline Aspidoras spilotus ${ }^{33}$ Nijssen \& Isbrücker, 1976 & & $\mathrm{X}^{(\mathrm{E})}$ & & & & 2 & Endemic \\
\hline Corydoras garbei ${ }^{34}$ Ihering, 1911 & & & & & $X^{(E)}$ & & Endemic \\
\hline \multicolumn{8}{|l|}{ Loricariidae } \\
\hline Hypostomus carvalhoi ${ }^{35}$ (Miranda Ribeiro, 1937) & & & & $X^{(\mathrm{E})}$ & & 1 & Endemic \\
\hline Hypostomus jaguribensis ${ }^{36}$ (Fowler, 1915) & & & & $X^{(\mathrm{E})}$ & & 1 & Endemic \\
\hline Hypostomus johnii ${ }^{37}$ (Steindachner, 1877) & $\mathrm{X}^{(\mathrm{E})}$ & & & & & & Endemic \\
\hline Hypostomus pusarum ${ }^{38}$ (Starks, 1913) & & & & & $\mathrm{X}$ & & Endemic \\
\hline Hypostomus sp. ${ }^{39}$ & $\mathrm{X}$ & & & & & & Native \\
\hline
\end{tabular}

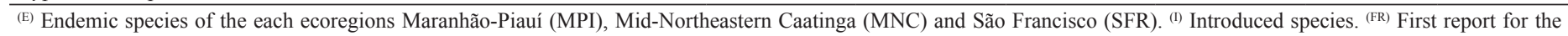
ecoregion. The number of each species represented the order in Appendix. 
Table 2. Continued...

\begin{tabular}{|c|c|c|c|c|c|c|c|}
\hline \multirow[b]{2}{*}{ Taxon } & \multicolumn{3}{|c|}{ Ibiapaba } & \multicolumn{2}{|c|}{ Araripe } & \multirow[b]{2}{*}{$\mathbf{C U}$} & \multirow[b]{2}{*}{$\begin{array}{c}\text { Status } \\
\text { Caatinga }\end{array}$} \\
\hline & $\begin{array}{c}\text { Parnaíba } \\
\text { (MPI) }\end{array}$ & $\begin{array}{l}\text { Coreaú } \\
\text { (MNC) }\end{array}$ & $\begin{array}{c}\text { Timonha } \\
\text { (MNC) }\end{array}$ & $\begin{array}{c}\text { Jaguaribe } \\
\text { (MNC) }\end{array}$ & $\begin{array}{c}\text { São } \\
\text { Francisco } \\
\text { (SFR) } \\
\end{array}$ & & \\
\hline Parotocinclus cearensis ${ }^{40}$ Garavello, 1977 & $\mathrm{X}$ & $\mathrm{X}$ & & $\mathrm{X}$ & & 1,2 & Endemic \\
\hline Parotocinclus haroldoi ${ }^{41}$ Garavello, 1988 & & & $\mathrm{X}^{(\mathrm{FR})}$ & & & 3 & Endemic \\
\hline Parotocinclus jumbo ${ }^{42}$ Britski \& Garavello, 2002 & & & & $\mathrm{X}^{(\mathrm{E})}$ & & 1 & Endemic \\
\hline Parotocinclus sp. ${ }^{43}$ & & & & $\mathrm{X}$ & & 1 & Native \\
\hline \multicolumn{8}{|l|}{ Heptapteridae } \\
\hline Pimelodella parnahybae ${ }^{44}$ Fowler, 1941 & $\mathrm{X}^{(\mathrm{E})}$ & & & & & & Endemic \\
\hline Rhamdia quelen ${ }^{45}$ (Quoy \& Gaimard, 1824) & & & & $\mathrm{X}$ & $\mathrm{X}$ & 1 & Native \\
\hline \multicolumn{8}{|l|}{ Auchenipteridae } \\
\hline Trachelyopterus galeatus ${ }^{46}$ (Linnaeus, 1766) & & & & $\mathrm{X}$ & & 1 & Native \\
\hline Trachycorystes cf. cratensis $^{47}$ (Miranda Ribeiro, 1937) & & & & $X^{(\mathrm{E})}$ & & 1 & Endemic \\
\hline \multicolumn{8}{|l|}{ CYPRINODONTIFORMES } \\
\hline \multicolumn{8}{|l|}{ Poeciliidae } \\
\hline Poecilia reticulata ${ }^{48}$ Peters, 1859 & $\mathrm{X}^{(\mathrm{I})}$ & $\mathrm{X}^{(\mathrm{I})}$ & & $\mathrm{X}^{(\mathrm{I})}$ & $\mathrm{X}^{(\mathrm{I})}$ & 1,2 & Introduced \\
\hline Poecilia vivipara ${ }^{49}$ Bloch \& Schneider, 1801 & $\mathrm{X}$ & $\mathrm{X}$ & & & $\mathrm{X}$ & 2 & Native \\
\hline Poecilia sp. $^{50}$ & & & $\mathrm{X}$ & & & 3 & Native \\
\hline Xiphophorus helleri ${ }^{51}$ Heckel, 1848 & & & & $\mathrm{X}^{(\mathrm{I})}$ & & 1 & Introduced \\
\hline \multicolumn{8}{|l|}{ SYNBRANCHIFORMES } \\
\hline \multicolumn{8}{|l|}{ Synbranchidae } \\
\hline Synbranchus marmoratus ${ }^{52}$ Bloch, 1795 & & $\mathrm{X}$ & $\mathrm{X}$ & & & 2,3 & Native \\
\hline \multicolumn{8}{|l|}{ PERCIFORMES } \\
\hline \multicolumn{8}{|l|}{ Cichlidae } \\
\hline Cichla sp..$^{53}$ Agassiz, 1831 & & $\mathrm{X}^{(\mathrm{I})}$ & & & & & Introduced \\
\hline Cichlasoma orientale $^{54}$ Kullander, 1983 & & $\mathrm{X}$ & $\mathrm{X}$ & $\mathrm{X}$ & & $1,2,3$ & Endemic \\
\hline Cichlasoma sanctifranciscense $e^{55}$ Kullander, 1983 & $\mathrm{X}$ & & & & & & Endemic \\
\hline Crenicichla menezesi $i^{56}$ Ploeg, 1991 & $\mathrm{X}$ & $\mathrm{X}$ & $\mathrm{X}$ & & & 3 & Native \\
\hline Geophagus brasiliensis $^{57}$ (Quoy \& Gaimard, 1824) & & & & $\mathrm{X}$ & $\mathrm{X}$ & 1 & Native \\
\hline Oreochromis niloticus ${ }^{58}$ (Linnaeus, 1758) & & $\mathrm{X}^{(\mathrm{I})}$ & & $X^{(1)}$ & $\mathrm{X}^{(\mathrm{I})}$ & 1 & Introduced \\
\hline Coptodon rendalli ${ }^{59}$ (Boulenger, 1897) & & & & & $\mathrm{X}^{(\mathrm{I})}$ & & Introduced \\
\hline Total endemic species & 5 & 1 & 0 & 5 & 4 & 11 & 29 \\
\hline Total exotic species & 1 & 3 & 0 & 3 & 3 & 3 & 5 \\
\hline Total species & 26 & 16 & 11 & 20 & 25 & 27 & 59 \\
\hline
\end{tabular}

(E) Endemic species of the each ecoregions Maranhão-Piauí (MPI), Mid-Northeastern Caatinga (MNC) and São Francisco (SFR). ${ }^{\left({ }^{(1)}\right.}$ Introduced species. ${ }^{(\mathrm{FR})}$ First report for the ecoregion. The number of each species represented the order in Appendix.

\section{Expansion of distribution}

In this study, Parotocinclus haroldoi is for the first time reported for the Mid-Northeastern Caatinga ecoregion based on individuals captured in Passagem, a stream in the Timonha river basin (Granja, Ceará State). A small detritivorous catfish, $P$. haroldoi lives in small groups, with a preference for shallow streams with clear or slightly turbid water and a substratum of rocks, twigs, sand and mud. The present report expands the distribution of the species, previously considered endemic to the Maranhão-Piauí ecoregion (Ramos et al. 2014), although the river mouth of the Timonha basin is close to the small coastal basins of the Maranhão-Piauí ecoregion (Figure 1). The occurrence of the species in this stream may be explained by the geomorphological slope northwest of Ibiapaba, which may have formed as a result of headwater capture during intense neotectonic activity in the region (Claudino-Sales \& Lira 2011) or during periods of marine regression in the Pleistocene when the basins were connected. The new occurrence of Poeciliidae (Poecilia sp.) was registered in the only basin (Timonha) where the exotic species $P$. reticulata was not observed. Despite the limited sampling in this basin (a single point), 11 species were registered, three (27.2\%) of which endemic to the Caatinga (Table 2).

\section{Conservation perspectives}

Approximately $38 \%(\mathrm{n}=17)$ of the sampling points were located inside conservation units (Table 1). These points accounted for $45.7 \%$ $(n=27)$ of the captured species. Eleven of these species $(37.9 \%)$ were considered endemic to the Caatinga (Table 3). Chapada do Araripe EPA harbored the greatest number of species $(n=20)$, corresponding to $51.5 \%$ of all non-introduced species registered in Araripe, nine (45.0\%) of which were endemic to the Caatinga. The two CUs in Ibiapaba accounted for 16 species (48.4\% of all non-introduced species registered in Ibiapaba), six (11.1\%) of which were endemic to the Caatinga. More specifically, the Serra da Ibiapaba EPA yielded 11 species, of which four were endemic and seven were native (none were introduced), whereas the Ubajara NP yielded nine species, of which four were endemic, four were native and one was introduced (Table 3).

Interestingly, seven of the endemic (Caatinga) species captured in the three conservation units were observed in one unit only (in Chapada do Araripe EPA = Aspidoras menezesi, Hypostomus carvalhoi, Hypostomus jaguaribensis, Parotocinclus jumbo and Trachycorystes cf. cratensis; in Ubajara NP = Aspidoras spilotus; in Serra da Ibiapaba EPA = Parotocinclus haroldoi), while only two species occurred in all three units: Characidium 
Table 3 .Total number of endemic, native and introduced species of the Caatinga sampled in the tablelands of Araripe and Ibiapaba and in the three conservation units (CU) (1=Chapada do Araripe EPA; 2=Ubajara NP; 3=Serra da Ibiapaba EPA), with an indication of the number of units in which each was observed.

\begin{tabular}{lcccccccc}
\hline & Total & CU 1 & CU 2 & CU 3 & In 1 CU & In 2 CUs & In 3 CUs & CUs [\% total] \\
\hline Endemic & 29 & 9 & 4 & 4 & 7 & 2 & 2 & $11[37.9 \%]$ \\
Native & 25 & 8 & 4 & 7 & 8 & 4 & 1 \\
Introduced & 5 & 3 & 1 & 0 & 2 & 1 & 0 \\
\hline
\end{tabular}

bimaculatum and Cichlasoma orientale (Table 3). In general, the conservation units in the study area protected a relatively small proportion of the total number of species, and even fewer endemic species, whereas more than $60 \%$ of the introduced species were observed at sampling points inside the units (Table 3). The low representativeness of native and endemic species inside these units may be explained by inadequate CU design: most of the headwaters streams which flow through the units are located in urbanized areas where they are exposed to adverse environmental impacts (removal of riparian vegetation, contamination with agricultural pesticides and fertilizers, etc.) and possibly the introduction of non-native species (Blackburn et al. 2015).

The highland forest enclaves of Northeastern Brazil not only represent important remnants of rain forest (Tabarelli et al. 2005, Santos et al. 2007), but boast a highly endemic fauna and flora. The enclaves in Borborema (Pernambuco and Paraíba) have been comprehensively surveyed, revealing high levels of endemism for bromeliads ( $33 \%$ in a single enclave), birds $(13 \%)$ and mammals (7\%) (Siqueira-Filho 2004, Souza et al. 2004). Some of these species have a limited geographical distribution and are endemic to the Caatinga, making them more vulnerable to extinction, especially when populations are small (Gaston et al. 1998). The swift and relentless degradation of the Caatinga and the processes of desertification and climate change which threaten the local biodiversity highlight the need for greater efforts at protecting both terrestrial and aquatic ecosystems (Maltchik \& Medeiros 2012).

The present study provides new information on the fish assemblages of humid highland forest enclaves in two tablelands (Araripe and Ibiapaba) in semiarid Northeastern Brazil which serve as drainage divides between the ecoregions Maranhão-Piauí (Parnaíba river basin), Mid-Northeastern Caatinga (Timonha, Coreaú and Jaguaribe river basins) and São Francisco. Nearly half the sampled species (29/59) were considered endemic to the Caatinga, and five were introduced. Only 11 of the species endemic to the Caatinga were captured in conservation units, but it should be kept in mind that the study was not specifically designed to evaluate the diversity of these units (with the exception of Ubajara NP). Most sampling points inside the units were located in basins of the Mid-Northeastern Caatinga ecoregion ( $n=16 ; 94.1 \%$ ). Thus, further studies are necessary to determine the ability of these units to protect the fish fauna of the Caatinga, especially in the São Francisco and Parnaíba river basins.

In addition, the distribution of $P$. haroldo $i$ was expanded to include the Timonha river basin in the westernmost reaches of the Mid-Northeastern Caatinga ecoregion. The fact that all samplings were carried out in the rainy season leaves unanswered questions about the impact of drought periods on the dynamics of the fish fauna in these ecosystems. According to Rosa \& Groth (2004), humid highland forest enclaves serve as permanent refuges. Future research may shed light on the role of these ecosystems in the maintenance of the local and regional fish fauna.

The diversity of endemic species and the singularity of these highland enclaves justify the creation of new conservation units (or the expansion of existing ones) in order to include important areas of aquatic ecosystems, such as riverheads, thereby reducing upstream anthropic impacts.

The present study provides new taxonomic information to subsidize future investigations into such fields as biogeography, ecology, conservation, bioinvasion and macroecology, and evidences the need for greater efforts to protect the highly endemic fish fauna of the highland forest enclaves of Northeastern Brazil.

\section{Appendix}

List of vouchers of the Universidade Federal do Rio Grande do Norte (UFRN) and the Universidade Federal da Paraíba (UFPB). The numbers refer to the numbering of each species in Table 2.

${ }^{1}$ (UFPB 7154), ${ }^{2}$ (UFPB 7108), ${ }^{3}$ (UFPB 7146, UFPB 7160), ${ }^{4}$ (UFRN 357, UFRN 1884, UFRN 1593), ${ }^{5}$ (UFPB 7150), ${ }^{6}$ (UFPB 9258), ${ }^{7}$ (UFPB 9259), ${ }^{8}$ (UFPB 9256, UFRN 1320, UFRN 1512, UFRN 1675), ${ }^{9}$ (UFPB 7103 , UFPB 9254, UFRN 358, UFRN 1195), ${ }^{10}$ (UFRN 1879, UFPB 7102, UFRN 1193), ${ }^{11}$ (UFPB 7103), ${ }^{12}$ (UFPB 7102, UFPB 7156), ${ }^{13}$ (UFPB 10612), ${ }^{14}$ (UFPB 10610), ${ }^{15}$ (UFPB 10616), ${ }^{16}$ (UFPB 10611), ${ }^{17}$ (UFPB 10615), ${ }^{18}$ (UFPB 7101), ${ }^{19}$ (UFPB 6751), ${ }^{20}$ (UFRN 2511), ${ }^{21}$ (UFPB 7111), ${ }^{22}$ (UFPB 7110, UFRN 1677, UFRN 1883), ${ }^{23}$ (UFRN 2677), ${ }^{24}$ (UFPB 6750), ${ }^{25}$ (UFPB 9269), ${ }^{26}$ (UFPB 10614), ${ }^{27}$ (UFPB 6754, UFPB 7100, UFPB 7117, UFPB 9275, UFRN 348, UFRN 604, UFRN 1261, UFRN 1325, UFRN 2684), ${ }^{28}$ (UFPB 6760), ${ }^{29}$ (UFPB 9427, UFRN 1521, UFRN 1580), ${ }^{30}$ (UFPB 9415), ${ }^{31}$ (UFRN 1879), ${ }^{32}$ (UFPB 7140), ${ }^{33}$ (UFRN 1466), ${ }^{34}$ (UFRN 2685), ${ }^{35}$ (UFRN 1810), ${ }^{36}$ (UFRN 352, UFRN 359, UFRN 1506), ${ }^{37}$ (UFPB 9267) ${ }^{38}$ (UFPB 7115), ${ }^{39}$ (UFPB 9265), ${ }^{40}$ (UFPB 9248, UFRN 1505), ${ }^{41}$ (UFRN 1294), ${ }^{42}$ (UFRN 350), ${ }^{43}$ (UFRN 1250), ${ }^{44}$ (UFPB 9279), ${ }^{45}$ (UFRN 1201),${ }^{46}$ (UFPB 9286), ${ }^{47}$ (UFRN 356), ${ }^{48}$ (UFPB 6755, UFRN 1476), ${ }^{49}$ (UFPB 9252), ${ }^{50}$ (UFRN 1468), ${ }^{51}$ (UFRN 1259), ${ }^{52}$ (UFPB 9280), ${ }^{53}$ (UFPB 9263), ${ }^{54}$ (UFPB 9249, UFRN 361), ${ }^{55}$ (UFPB 9260), ${ }^{56}$ (UFRN 943, UFPB 9278), ${ }^{57}$ (UFPB 7099, UFRN 589), ${ }^{58}$ (UFPB 7116, UFRN 355), ${ }^{59}$ (UFRN 2512).

\section{Acknowledgments}

We would like to thank the staff of the Laboratório de Ecologia Aquática of the Universidade Federal do Ceará and Laboratório de Ictiologia Sistemática e Evolutiva of the Universidade Federal do Rio Grande do Norte. The study was funded by CNPq/PPBIO proposal \#457463/2012-0 and CNPq/ICMBio proposal \#552009/2011-3 and \#552086/2011-8.

\section{References}

ABELL, R., THIEME, M.L., REVENGA, C., BRYER, M., KOTTELAT, M., BOGUTSKAYA, N., COAD, B., MANDRAK, N., CONTRERAS-BALDERAS, S., BUSSING, W., STIASSNY, M.L.J., SKELTON, P., ALLEN, G.R., UNMACK, P., NASEKA, A., NG, R., SINDORF, N., ROBERTSON, J., ARMIJO, E., HIGGINS, J.V., HEIBEL, T.J., WIKRAMANAYAKE, E., OLSON, D., LÓPEZ, H.L., REIS, R.E., LUNDBERG, J.G., SABAJ-PÉREZ, M.H. \& PETRY, P. 2008. Freshwater ecoregions of the world: a new map of biogeographic units for freshwater biodiversity conservation. Bioscience 58(5):403-414.

AB'SÁBER, A.N. 2003. Os domínios de natureza no Brasil - potencialidades paisagísticas. Ateliê Editorial, São Paulo.

ALBERT, J.S., PETRY, P. \& REIS, R.E. 2011. Major Biogeographic and Phylogenetic Patterns. In Historical biogeography of Neotropical freshwater fishes (J.S. ALBERT \& R.E. REIS, eds.). University of California Press, Berkeley, p.21-57

ANDRADE-LIMA, D. 1982. Present day forest refuges in Northeastern Brazil. In Biological Diversification in the Tropics (G.T. Prance, ed.). Columbia University Press, New York, p.245-254. 
BLACKBURN, TM., LOCKWOOD, JL. \& CASSEY, P. 2015. The Influence of numbers on invasion sucess. Mol. Ecol. 24(9):1942-1953.

BOTERO, J.I.S., GARCEZ, D.S., CASCON, P., LIMA, S.M.Q., BRITO, L.B.M. \& ROBERTO, I.J. 2014. Anfíbios e peixes do Parque Nacional de Ubajara e entorno. UFC/LABOMAR/NAVE, Fortaleza.

CLAUDINO-SALES, L. \& LIRA, M.V. 2011. Megageomorfologia do nordeste do estado do Ceará, Brasil. Caminhos de Geografia 12(38):200-209.

GASTON, K. J. 1998. Rarity as double jeopardy. Nature 394:229-230.

LANGEANI, F.L., BUCKUP, P.A., MALABARBA, L.R., PY-DANIEL, L.H.R., LUCENA, C.A., ROSA, R.S., ZUANON, J.A.S., LUCENA, Z.M.S., BRITTO, M.R., OYAKAWA, O.T. \& GOMES-FILHO, G. 2009. Peixes de água doce. In Estado da arte e perspectivas para a Zoologia no Brasil (R.M. Rocha \& W.A.P. Boeger, eds.). Editora UFPR, Curitiba, p.211-230.

LÉVÊQUE, C., OBERDORFF, T., PAUGY, D., STIASSNY, M.L.J. \& TEDESCO, P.A. 2008. Global diversity of fish (Pisces) in freshwater. Hydrobiologia 595:545-567.

MALTCHIK, L. \& MEDEIROS, E.S.F. 2012. Conservation importance of semiarid streams in north-eastern Brazil: implications of hydrological disturbance and species diversity. Aquat. Conser. Mar. Freshw. Ecosyst. 16(7):665-677.

MEDEIROS, E.S.F. \& MALTCHIK, L. 2001. Fish assemblage stability in an intermittently flowing stream from the Brazilian semiarid region. Austral Ecol. 26(2):156-164.

Ministério do Meio Ambiente (MMA) Brasil. 2014. Lista nacional oficial de espécies da fauna ameaçadas de extinção. Portaria Nº445, 17 de dezembro 2014.

Ministério de Minas e Energia (MME) Brasil. 1996. Projeto avaliação hidrogeológica da bacia sedimentar do Araripe. Ministério de Minas e Energia, Recife.

MIRANDA-RIBEIRO, A. 1937. Sobre uma collecção de vertebrados do nordeste brasileiro. Primeira parte: peixes e batrachios. In O campo (A. MIRANDARIBEIRO, eds). Rio de Janeiro, p.54-56.

NIJSSEN, H. \& ISBRUCKER, I.J.H. 1976. The South American plated catfish genus Aspidoras R. Von Ihering, 1907. Bijadragem tot de Dierkunde. 46(1): 107-131

RAMOS, T.P.A., RAMOS, R.T.C. \& RAMOS, S.A.Q.A. 2014. Ichthyofauna of the Parnaíba river Basin, Northeastern Brazil. Biota Neotrop. 14(1):1-8. http:// dx.doi.org/10.1590/S1676-06020140039 (último acesso em 10/12/2015).

REIS, R.E., KULLANDER, S.O. \& FERRARIS Jr., C.J. 2003. Check List of the freshwater fishes of South and Central America. EDIPUCRS, Porto Alegre.

ROSA, R.S. \& GROTH, F. 2004. Ictiofauna dos ecossistemas de brejos de altitude de Pernambuco e Paraíba. In Brejos de altitude em Pernambuco e Paraíba (K.C. PÔRTO, J.J.P. CABRAL \& M. TABARELLI, eds). Ministério do Meio Ambiente, Brasília, p.201-228.
ROSA, R.S., MENEZES, N.A., BRITSKI, H.A., COSTA, W.J.E. \& GROTH, F. 2003. Diversidade, padrões de distribuição e conservação dos peixes da Caatinga. In Ecologia e conservação da Caatinga (I.R. LEAL, M. TABARELLI \& J.M.C. DA SILVA, eds). Editora Universitária UFPE, Recife, p.135-180.

SÁ, I.B., RICHÉ, G.R. \& FOTIUS, G.A. 2004. As paisagens e o processo de degradação do semi-árido nordestino. In Biodiversidade da Caatinga: áreas e ações prioritárias para a conservação (J.M.C. SILVA, M. TABARELLI, M.T. FONSECA \& L.V. LINS, eds). Ministério do Meio Ambiente, Brasília, p.17-36.

SANTOS, A.M.M., CAVALCANTI, D.R., DA SILVA, J.M.C. \& TABARELLI, M. 2007. Biogeographical relationships among tropical forests in north-eastern Brazil. J. Biogeogr. 34:437-446.

SANTOS, J.C., LEAL, I.R., ALMEIDA-CORTEZ, J.S., FERNANDES, G.W. \& TABARELLI, M. 2011. Caatinga: the scientific negligence experienced by a dry tropical forest. Trop. Conserv. Sci. 4(3):276-286.

SIQUEIRA-FILHO, J.A. 2004. As bromélias do estado de Pernambuco: Diversidade e status de conservação. In Brejos de Altitude em Pernambuco e Paraíba (K.C. PÔRTO, J.J.P. CABRAL \& M. TABARELLI, eds). Ministério do Meio Ambiente, Brasília, p.99-110.

SOUZA, M.A.N., LANGUTH, A. \& AMARAL, E.G. 2004. Mamíferos dos Brejos de Altitude de Paraíba e Pernambuco. In Brejos de Altitude em Pernambuco e Paraíba (K.C. PÔRTO, J.J.P. CABRAL \& M. TABARELLI, eds). Ministério do Meio Ambiente, Brasília, p.229-254.

Secretaria de Recursos Hídricos do Estado do Ceará (SRH). 2005. Implantação do sistema de monitoramento/gestão de uma área piloto do aquífero Missão Velha, na Bacia Sedimentar do Araripe. SRH, Fortaleza.

TABARELLI, M. \& SANTOS, A.M.M. 2004. Uma breve descrição sobre a história natural dos brejos nordestinos. In Brejos de Altitude em Pernambuco e Paraíba (K.C. PÔRTO, J.J.P. CABRAL \& M. TABARELLI, eds). Ministério do Meio Ambiente, Brasília, p.17-24.

TABARELLI, M., PINTO, L.P., SILVA, M.C., HIROTA, M. \& BEDÊ, L. 2005. Challenges and opportunities for biodiversity conservation in the Brazilian Atlantic Forest. Conserv. Biol. (19):695-700.

VITULE, J.R.S., FREIRE, C.A. \& SIMBERLOFF, D. 2009. Introduction of nonnative freshwater fish can certainly be bad. Fish Fish. (10):98-108.

VILLÉGER, S., GRENOUILLET, G. \& BROSSE, S. 2014. Functional homogenization exceeds taxonomic homogenization among European fish assemblages. Global Ecol. Biogeogr. (23):1450-1460.

Received: 09/10/2016

Accepted: 14/10/2016 